\title{
REAC technology as optimizer of stallion spermatozoa liquid storage
}

\author{
Fiammetta Berlinguer ${ }^{1 \dagger}$, Valeria Pasciu', Sara Succu', Ignazio Cossu², Sabrina Caggiu', Daniela Addis ${ }^{1}$, \\ Alessandro Castagna ${ }^{3}$, Vania Fontani $i^{3,4}$, Salvatore Rinaldi ${ }^{3,4^{*}}$ (i) and Eraldo Sanna Passino ${ }^{1+}$
}

\begin{abstract}
Background: REAC technology (acronym for Radio Electric Asymmetric Conveyor) is a technology platform for neuro and bio modulation. It has already proven to optimize the ions fluxes at the molecular level and the molecular mechanisms driving cellular asymmetry and polarization.

Methods: This study was designed to verify whether this technology could extend spermatozoa life-span during liquid storage, while preserving their functions, DNA integrity and oxidative status. At $0,24,48$, and $72 \mathrm{~h}$. of storage at $4{ }^{\circ} \mathrm{C}$, a battery of analyses was performed to assess spermatozoa viability, motility parameters, acrosome status, and DNA integrity during REAC treatment. Spermatozoa oxidative status was assessed by determining lipid peroxidation, the activity of superoxide dismutase (SOD), and the total antioxidant capacity.

Results: During liquid storage REAC treated spermatozoa, while not showing an increased viability nor motility compared to untreated ones, had a higher acrosome $(p>0.001)$ and DNA integrity $(p>0.01)$. Moreover, the analysis of the oxidative status indicated that the mean activity of the intracellular superoxide dismutase (SOD) was significantly higher in REAC treated spermatozoa compared to untreated controls $(p<0.05)$, while the intracellular concentration of malondialdehyde (MDA), an end product of lipid peroxidation, at the end of the REAC treatment was higher in untreated controls $(p>0.05)$. The REAC efficacy on spermatozoa oxidative status was also evidenced by the higher trolox equivalent antioxidant capacity (TEAC) found in both the cellular extract $(p<0.05)$ and the storage media of REAC treated spermatozoa compared to untreated controls $(p<0.0001)$.

Conclusion: The present study demonstrated that REAC treatment during liquid storage preserves spermatozoa acrosome membrane and DNA integrity, likely due to the enhancement of sperm antioxidant defenses. These results open new perspective about the extending of spermatozoa functions in vitro and the clinical management of male infertility.
\end{abstract}

\section{Background}

Radio Electric Asymmetric Conveyer Technology for therapeutic use (REAC) is a recent technology whose aim is to optimize the ion fluxes at the molecular level, and to concentrate all the micro currents produced by these ion fluxes through the asymmetric conveyer-probe to optimize the molecular mechanisms driving cellular asymmetry and polarization. In fact, it is able to properly asymmetrically convey the ion currents, "re-setting" the altered bioelectric

\footnotetext{
* Correspondence: srinaldi@irf.it

${ }^{\dagger}$ Equal contributors

${ }^{3}$ Departments of Regenerative Medicine, Rinaldi Fontani Institute, Viale Belfiore 43, 50144 Florence, Italy

${ }^{4}$ Research Department, Rinaldi Fontani Foundation, Viale Belfiore 43, 50144 Florence, Italy

Full list of author information is available at the end of the article
}

fields and therefore recovering the correct cell electrometabolic activity which drives the migration, proliferation and differentiation of a host of cell types and promotes reparative and regenerative process of tissues [1-8].

Sperm cells are polarized cells [9]. Development, survival, and effectiveness of sperm are closely linked to the phenomena of cell polarity. On these bases, we checked whether REAC technology is effective in extending and maintaining sperm functions and DNA integrity during liquid storage at $4{ }^{\circ} \mathrm{C}$. In fact, sperm cryopreservation and sperm liquid storage by short-term refrigeration at $+4{ }^{\circ} \mathrm{C}$ lead to a significant decline in sperm quality [10]. In particular, during sperm liquid storage, we observe a deterioration of sperm functions also followed by DNA damage [11]. 
Oxidative stress has been proposed to be a major factor involved in sub lethal cryodamage of sperm in many species, including horses [12, 13]. After semen dilution and cryopreservation, there is a significant reduction in the level of spermatozoa antioxidants, thus leading to an enhanced susceptibility of these cells to peroxidative injuries. In addition, semen cryopreservation is associated with increased generation of reactive oxygen species (ROS) [14, 15]. Spermatozoa are vulnerable to oxidative attack because they contain an abundance of polyunsaturated fatty acids that are susceptible to lipid peroxidation. Functionally important proteins and DNA are also subject to oxidative modification and adduction by aldehydes, generated as a consequence of the peroxidative process [16]. Oxidative stress ultimately triggers the intrinsic apoptotic pathway, leading to a rapid loss of motility and cell death. As suggested by Riel et al. [17], the iatrogenic damage caused by semen dilution and cryopreservation may be also due to the presence of ions in the surrounding medium coupled with sperm membrane changes occurring during storage at non-physiological temperatures. Sodium-potassium-dependent ATPase, which regulates proper intracellular concentration of sodium, is highly sensitive to hypothermia. At low temperatures, reduction of its activity impairs proper functioning of the sodium pump, resulting in uptake of sodium ions from extracellular milieu. Sodium ions were shown to be detrimental to the nucleus and DNA $[18,19]$. In addition, a perturbation of ion flows can result in alterations of plasma and mitochondrial membranes resting potential and thus of cell functions. Plasma membrane resting potential arises from the combined action of ion channels and ion pumps, and it regulates cell-autonomous properties such as proliferation, differentiation and apoptosis in mature somatic cells as well as stem cells [20]. The loss of mitochondrial membrane potential has been associated with a significant increase in ROS generation [21, 22]. All these phenomena are related to alterations of cell polarity and may be responsible for hampering cell preservation at low temperatures.

Thus, considering the abovementioned REAC treatment effects, this study was designed to test whether this technology may be used to extend spermatozoa lifespan and functions during liquid storage at $4{ }^{\circ} \mathrm{C}$. Since spermatozoa are particularly vulnerable to oxidative attack, the effect of this technology on sperm cell oxidative status during liquid storage was also studied. In the present study, we choose the stallion as experimental model because REAC protocol of Veterinary Neuro Psycho Physical Optimization (VNPPO) in vivo has proven to exert a positive effect in the semen quality of idiopathic subfertile stallions [23].

\section{Methods}

\section{Chemicals}

All chemicals in this study were purchased from Sigma Chemical CO. (St. Louis, MO, USA), unless stated otherwise.

\section{Description of Radio Electric Asymmetric Conveyer (REAC) Technology}

Radio Electric Asymmetric Conveyer Technology (REAC) is a technological platform for bio and neuro modulation. A detailed description of REAC mechanism of action can be found in Maioli 2016 [2]. Briefly, REAC is an asymmetric technology, because a normal electric circuit has two physical poles: one positive and one negative (symmetrical circuit); in the REAC technology, there is only one single physical pole (asymmetrical circuit). This pole becomes the attractor (Asymmetric Conveyer) for the currents induced in the body by the radio frequency emission. This scheme has been developed to create an asymmetric circuit for better interact with the asymmetric mechanism underlying the cell polarity [24], in order to optimize its functions. In fact, REAC technology is able to modulate the current flows existing both at cellular and body level, when these are altered. Another peculiarity of REAC Technology is the low power level used in radio frequency emission. This is necessary to induce current flows of intensity comparable with those of cell polarity. Higher power levels would disturb the adjustment mechanisms of cell polarity. REAC devices use only two radio frequencies $(2.4$ and $5.8 \mathrm{GHz}$ ) because these are the most widely used and permitted at the international level. In this study, we used the biomodulation REAC-VIVSI treatment protocol. The REAC device used in this study was B.E.N.E (ASMED, Florence, Italy).

\section{Semen source and preparation}

The experimental procedures were carried out during horse breeding season (January-July). Semen collection was carried on at the Department of Stallion Reproduction of the Regional Agency for Research in Agriculture (AGRIS Sardegna, Ozieri, Sassari, Italy), while the analytical work was carried on at the laboratories of the Department of Veterinary Medicine of the University of Sassari (UNISS, Sassari, Italy). These facilities meet the requirements of the European Union for Scientific Procedure Establishments. Ejaculates were obtained by artificial vagina from eight adult stallions of different ages (from 12 to 24 years), and different breeds. In particular, stallions were identified with progressive number from 1 to 8 (stallion 1: Thoroughbred; stallions 2, 3, 4, 6: Arabian; stallions 5, 7, 8: Warmblood). The stallions were housed individually in boxes, and fed ad libitum with a diet containing adequate nutrients. At the moment of the study, they had been housed in AGRIS for over 5 years and used exclusively for breeding, and they are 
currently still housed at the same center. All evaluated stallions had a career in sports flat racing and show jumping.

The eight stallions enrolled in this study were used for breeding, and the frequency in semen collection during the entire breeding season was twice a week. We used for this study two ejaculates from each male, collected 15 days apart from each other. Semen was transported to the AGRIS laboratory within $5 \mathrm{~min}$ after collection, and it was immediately processed. Sperm concentration was evaluated by NucleocounterSp - 100 Chemometech. Thereafter, semen was diluted up to $50 \times 10^{6}$ spermatozoa/ $\mathrm{mL}$ with a commercial media for liquid storage (Kenney's extender, IMV Technologies). Ejaculates from each stallion were kept separated throughout all experimental procedures and, once diluted, they were placed in two separated Falcon tubes and transported to the UNISS laboratory under controlled temperature $\left(4^{\circ} \mathrm{C}\right)$ within $1 \mathrm{~h}$.

Upon arrival, one sample from each stallion was allocated to the treated and untreated group. The experimental groups were placed in two refrigerators at $4{ }^{\circ} \mathrm{C}$ and kept there for $72 \mathrm{~h}$. The REAC device was placed in the refrigerator where treated samples were stored, and it was set at $2.4 \mathrm{GHz}$, and its conveyer electrodes were immersed into the semen liquid storage media.

\section{Experimental design}

In order to evaluate the effects of REAC technology during stallion semen liquid storage, a battery of analyses was performed. The parameters analyzed included different semen molecular and cellular features, measured before and during REAC treatment, such as viability, motility parameters, acrosome status, and DNA integrity. In addition, the oxidative status was assessed by determining lipid peroxidation, the activity of superoxide dismutase (SOD), and the total antioxidant capacity. These analyses were performed on sub-samples of cooled semen collected at 0,24 , 48 , and $72 \mathrm{~h}$. after the beginning of the REAC treatment. Each analysis was replicated 3 times.

\section{Viability and motility parameters assessment}

In vitro viability was assessed by eosin-nigrosin stain. Briefly, the eosin-nigrosin solution was prepared as described by Pintado et al. [25]. Briefly, $10 \mathrm{~g}$ nigrosin was dissolved in distilled water by boiling, and filtered into a cylinder containing $0.7 \mathrm{~g}$ eosin, $7.5 \mathrm{ml}$ of $50 \mathrm{mmol}$ glucose $1-1$, and $7.5 \mathrm{ml}$ tartrate phosphate buffer (TPB) (50 mM Na2HPO4 1-1, 25 mM KH2PO4 l-1, $77 \mathrm{mM}$ potassium sodium tartrate $1-1$ ), and the volume made up to $100 \mathrm{~mL}$. The solution was kept at $5{ }^{\circ} \mathrm{C}$. Staining was carried out by mixing an aliquot of spermatozoa suspended in saline medium with eosin-nigrosin solution (1:3 dilution) for $30 \mathrm{~s}$ before preparing a smear and drying on a warm plate at $37{ }^{\circ} \mathrm{C}$. At least 200 cells were counted for each slide. Sperm motility parameters were assessed using a computer-assisted sperm analysis (CASA) system (Sperm Class Analyser, S.C.A. v 3.2.0, Microptic S.L., Barcelona, Spain) with setting of 25 frames acquired to avoid sperm track overlapping, minimum contrast 10 , minimum velocity of average path $30 \mu \mathrm{m} / \mathrm{s}$, progressive motility $>80 \%$ straightness. This system has a specific setup for stallion sperm evaluation. In particular, it was set up as follows: minimum contrast - 70; low and high static size gates $-0.6-4.32$; low and high intensity gates -0.20 1.92; low and high elongation gates 7-91; default cell size - 10 pixels; default cell intensity -80 . For each sample, $5 \mu \mathrm{L}$ subsample of sperm suspension was loaded into a pre-warmed analysis chamber with a depth of $10 \mu \mathrm{m}$ (Makler Counting chamber, Sefi-Medical Instruments ltd., Biosigma S.r.l., Italy) and a minimum of 500 sperms per subsample were analyzed in at least four different microscopic fields. Sperm motility was assessed at $37{ }^{\circ} \mathrm{C}$ at $40 \times$ using a phase contrast microscope. The parameters evaluated included: percentage of progressive motile spermatozoa (PM); percentage of rapid spermatozoa (rapid); average path velocity (VAP, $\mathrm{mm} / \mathrm{s}$; the average velocity of the smoothed cell path); curvilinear velocity (VCL, $\mathrm{mm} / \mathrm{s}$; the average velocity measured over the actual point to point track followed by the cell); straight-line velocity (VSL, $\mathrm{mm} / \mathrm{s}$; the average velocity measured in a straight line from the beginning to the end of the track); linearity index (LIN, \%; the average value of the ratio VSL/VCL); straightness index (STR, $\%$; the average value of the ratio VSL/VAP); amplitude of lateral head displacement $(\mathrm{ALH}, \mathrm{mm}$; the mean width of the head oscillation as the sperm swim); beat cross-frequency (BCF, Hz; the frequency of sperm head crossing the average path in either direction); wobble (WOB; VAP/VCL $\times 100$, \%; a measure of the oscillation of the actual trajectory about its spatial average path).

\section{Acrosome integrity}

Acrosome integrity was evaluated by incubating spermatozoa with fluorescein isothiocynatelabeled PisumSativum agglutinin (FITC-PSA). The aliquots of sperm suspension were incubated for $15 \mathrm{~min}$ at $39{ }^{\circ} \mathrm{C}$ with FITC-PSA (5 $\mu \mathrm{g}$ / $\mathrm{mL}$ in phosphate buffered saline [PBS], $\mathrm{pH} 7.4$ ), and propidium iodide (PI; $14 \mu \mathrm{g} / \mathrm{mL}$ in phosphate buffered saline [PBS], pH 7.4). In order to reduce background fluorescence, unbound PSA and PI were removed by adding $200 \mu \mathrm{L}$ of PBS and spermatozoa were washed by centrifugation in a micro centrifuge at $800 \mathrm{~g}$ for $2 \mathrm{~min}$. The supernatant was aspirated and the pellet re-suspended in $100 \mu \mathrm{L}$ of PBS. After washing, a $10 \mu \mathrm{L}$ sample was put on a slide and cover slipped. The slide was immediately dried by leaving at $37^{\circ} \mathrm{C}$ for $10 \mathrm{~min}$ for immobilization of sperm cells. To evaluate the stained sperm cells, at least 200 cells were counted in duplicate for each sample, using a Diaphot (Nikon, Japan) 
epifluorescence microscope. Spermatozoa with intact plasma membrane and intact acrosome were PI and FITCPSA negative (no fluorescent staining), those with intact plasma membrane and damaged acrosome were PI negative and FITC-PSA positive (emitting green fluorescence), those with damaged plasma membrane and intact acrosome were PI positive and FITC-PSA negative (emitting red fluorescence), and finally those with damaged plasma membrane and damaged acrosome were PI and FITC-PSA positive (emitting both green and red fluorescence).

\section{DNA integrity assessment}

DNA damage was assessed by single-cell gel electrophoresis (comet assay). Analysis of the shape and length of "comet" tail, just like the DNA content in the tail, gives an assessment of DNA damage. The neutral comet assay allows the detection of double-strand breaks by subjecting lysed cell nuclei to an electrophoretic field at neutral $\mathrm{pH}$ [26], here performed according to the method described by Sakkas et al.[27], with slight modifications. Briefly, sperm suspension $(30 \mu \mathrm{L})$ was diluted in low-melting-point agarose at $37^{\circ} \mathrm{C}(80 \mu \mathrm{L} ; 1 \% \mathrm{w} / \mathrm{v})$. A $100-\mu \mathrm{L}$ mixture of sperm-agarose was immediately pipetted onto $1 \% \mathrm{w} / \mathrm{v}$ normal-melting-point agarosecoated slides. Slides were immersed in ice-cold lysing solution $(2.5 \mathrm{M} \mathrm{NaCl}, 100 \mathrm{mM}$ EDTA, 10 mMTris, $1 \%$ Triton $\mathrm{X}$, and 10 mMdithiothreitol [DTT]; $\mathrm{pH}=10$ ) for $1 \mathrm{~h}$ at $4{ }^{\circ} \mathrm{C}$. Slides were then immersed in lysing solution supplemented with proteinase $\mathrm{K}(10 \mu \mathrm{g} / \mathrm{mL})$. Incubation was performed during $1 \mathrm{~h}$ at $37^{\circ} \mathrm{C}$. After this step, slides were rinsed in PBS and then placed in a horizontal electrophoresis tank filled with freshly prepared electrophoresis neutral buffer (Tris-acetate-EDTA [TAE], pH 7.3). Electrophoresis was performed at $10 \mathrm{~V}$ and $6 \mathrm{~mA}$ for $20 \mathrm{~min}$. Following electrophoresis, the slides were neutralized with Tris- $\mathrm{HCl}$ buffer ( $\mathrm{pH}$ 7.5) for $5 \mathrm{~min}$ and then fixed in methanol.

Slides were stained with propidium iodide (PI), mounted with a coverslip and analyzed under an epifluorescence microscope. Digital comet images were captured with an Olympus microscope equipped with a CCD camera and Olympus CellF software. Fifty comets were measured per replicate sample (i.e., slide circle) using Comet Score software (TriTek Corp., Sumerduck, Virginia, USA). Scored parameters included percentages of head and tail DNA (a measurement of the proportion of total DNA that is present in the comet head and tail).

Sample preparation for the oxidative parameters analysis Five $\mathrm{mL}$ of semen samples $\left(50 \times 10^{6}\right.$ spermatozoa $\left./ \mathrm{mL}\right)$ was centrifuged at $1500 \mathrm{~g}$ for $10 \mathrm{~min}$. The obtained pellets were treated for cellular extraction with PBS containing $0,1 \%$ Triton X-100 (500 $\mu \mathrm{L}$ of PBS-Triton X-100 $0,1 \%$ every $250 \times 10^{6}$ total spermatozoa). Malondialdehyde concentration (MDA) and superoxide dismutase (SOD) activity were assayed in cellular extracts, while trolox equivalent antioxidant capacity (TEAC) was determined in both cellular extracts and extracellular supernatants.

\section{Superoxide dismutase (SOD) activity}

SOD activity was measured enzymatically as a decrease of the XTT (3'-(1-[(Phenylamino)-carbonyl]-3,4-tetrazolium)-bis(4-methoxy-6-nitro) benzenesulphonic acid hydrate) reduction by superoxide anion generated by xanthine oxidase [28].
a) Xanthine $+\mathrm{O}_{2} \stackrel{\mathrm{XO}}{\rightarrow}$ uric acid $+\mathrm{O}_{2}{ }^{-}$
b) $\mathrm{O}_{2}^{-}+\mathrm{XTT}$ (detector) $\rightarrow$ reduced XTT
c) $2 \mathrm{O}^{-}+2 \mathrm{H}^{+} \stackrel{\mathrm{SOD}}{\rightarrow} \mathrm{H}_{2} \mathrm{O}_{2}+\mathrm{O}_{2}$

SOD activity was assessed as the competition between reaction $\mathrm{c}$ and $\mathrm{b}$ which is measured as a decrease of the rate of XTT reduced. The reaction mixture contained $40.5 \mathrm{mM}$ sodium phosphate buffer $\mathrm{pH} 7.8 ; 15 \mathrm{mM}$ xanthine; EDTA 12,5 mM; XTT $30 \mathrm{mM}$ and $50 \mu \mathrm{L}$ of sample to complete a final volume of $500 \mu \mathrm{L}$. The reaction was initiated by the addition of xantine oxidase (XO) $(0.15 \mathrm{mUI})$ and the absorbance change at $470 \mathrm{~nm}$ was monitored each minute for 3 min total with a Hitachi spectrophotometer (U-2000). The values of SOD in the samples were expressed in $\mathrm{U} / \mathrm{mL}$ and calculated using a standard curve $(0,065-0,8 \mathrm{U} / \mathrm{ml})$. One enzyme unit (IU) is defined as the amount of SOD capable of transforming 1.0 mmole/min of $\mathrm{O} 2 \bullet$ ᄀ

\section{Quantification of lipid peroxidation end products: malondialdehyde (MDA)}

MDA, one of the several low-molecular-weight endproducts of LPO, was evaluated by the TBARS assay using thiobarbituric acid and a spectrophotometric method according to the TBA test described by Spanier and Traylor [29], with some modifications. $100 \mu \mathrm{L}$ of each sample (cell extract and extracellular supernatant) were added to $100 \mu \mathrm{L}$ glacial acetic acid 33\%, $75 \mu \mathrm{L}$ SDS 10\%, $100 \mu \mathrm{L}$ Tris- $\mathrm{HCl} 50 \mathrm{mM} \mathrm{pH} \mathrm{7,4} \mathrm{and} 250 \mu \mathrm{L}$ TBA 0,75\%. The mixture was then incubated for $1 \mathrm{~h}$ at $100{ }^{\circ} \mathrm{C}$ and immediately cooled on ice. After 10 min $200 \mu \mathrm{L}$ of acetic acid 33\% were added and samples were centrifuged for $20 \mathrm{~min}$ at $7000 \mathrm{~g}$. The supernatant absorbance was then read with Thermo Electron Corporation Genesys 10UV spectrophotometer (Thermo Fisher Scientific, Rodano, Milano, Italy), at $535 \mathrm{~nm}$. The values of MDA in the samples were expressed in $\mu \mathrm{M}$ units and calculated using a standard curve.

\section{Trolox equivalent antioxidant capacity (TEAC)}

Cell extract and extracellular supernatant antioxidant capacity was determined using the method described by 
Re et al.[30], and modified by Lewinska et al.[31]. Briefly, a fresh solution was prepared by dissolving $19.5 \mathrm{mg}$ 2,20-azinobis (3- ethylbenzthiazoline -6-sulphonic acid [ABTS]) and $3.3 \mathrm{mg}$ potassium persulphate in $7 \mathrm{~mL}$ of $0.1 \mathrm{M}$ phosphate buffer, $\mathrm{pH}$ 7.4. This solution was stored in the dark for $12 \mathrm{~h}$ for completion of the reaction. ABTS solution was diluted (usually approximately 1:80) in $0.1 \mathrm{~mol} / \mathrm{L}$ phosphate buffer, $\mathrm{pH} 7.4$, to give an absorbance reading at $734 \mathrm{~nm}$ of 1.0. The absorbance of the mixture was measured twice in a spectrophotometer (ThermoElecrom Corporation Genesys 10 UV, Madison, Wisconsin, USA), at $734 \mathrm{~nm}, 3 \mathrm{~min}$ after mixing a sample with the ABTS $\bullet\urcorner^{\circ}$ solution. The extent of ABTS $\bullet$ ' bleaching is proportional to the activity of antioxidants in a given sample. The antioxidant capacity was expressed as TEAC, the concentration of trolox producing the same effect as the sample studied.

The values of TEAC in the samples were calculated using a standard curve (5-20 mMtrolox in a total volume of $550 \mathrm{~mL}$ ) and were expressed as mMTrolox equivalent for extracellular supernatant and nmoli of trolox equivalent/109 spermatozoa for cell extract.

\section{Statistical analyses}

Statistical analyses were performed using the statistical software program Statgraphic Centurion XV (version15.2.06 for Windows; Stat Point Technologies Inc., Warrenton, VA, USA), and a probability of $p<0.05$ was considered to be the minimum level of significance. Data are expressed as mean \pm S.E. Differences in sperm parameters between the experimental groups at the different time points (hours of treatment) were assessed by general lineal model where: $\mathrm{Y}$ $=\mu+$ hours of treatment + group + hours of treatment $\mathrm{x}$ group + stallion. Hours of treatment and group were considered fixed factors and stallion a random factor. The method used to discriminate between the means was Fisher's least-significant-difference (l.s.d.) procedure. The probabilities obtained by the l.s.d. test were corrected by Bonferroni's correction for multiple comparisons. Data were normally distributed (Shapiro Wilk W test: $P>0.05$ ).

\section{Results}

\section{Viability}

The storage conditions under which sperm cells were maintained proved to preserve spermatozoa viability for at least $72 \mathrm{~h}$. No difference was observed in the number of vital spermatozoa assed by eosin dye exclusion in REAC treated samples and untreated controls (Fig. 1). The male factor was indeed the only factor able to influence spermatozoa viability, as outlined by the significant difference observed among the eight stallions (Additional file 1: Figure S1, panel B).

\section{Kinetic parameters and straightness index}

In general, kinetic parameters decreased significantly during the $72 \mathrm{~h}$. of observation, and no significant differences were observed between REAC treated samples and untreated controls (Fig. 2). Interestingly, the straightness index values were significantly higher in REAC treated samples compared to untreated controls (Additional file 2: Figure S2, panel A and B; $p<0.001$ ).

\section{Acrosome membrane integrity}

Acrosome membrane integrity was better preserved in REAC treated spermatozoa compared to untreated controls, as demonstrated by the higher percentage of spermatozoa showing an intact acrosome after FITC-PSA

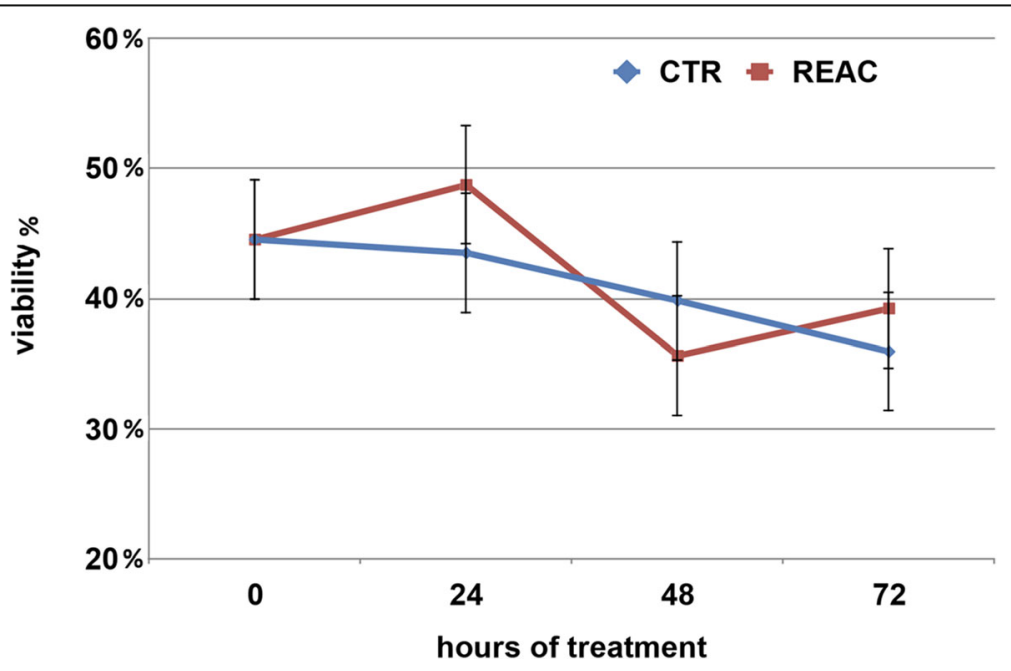

Fig. 1 Effect of REAC treatment on stallion spermatozoa viability, expressed expressed as percentage of viable cells over the total cells counted during $72 \mathrm{~h}$. of storage at $4{ }^{\circ} \mathrm{C}$. Values determined at the different time points are expressed as mean \pm S.E. A total of 16 ejaculates collected from eight stallions of different breeds (1: Thoroughbred; 2, 3, 4, 6: Arabian; 5, 7, 8: Warmblood) were used 


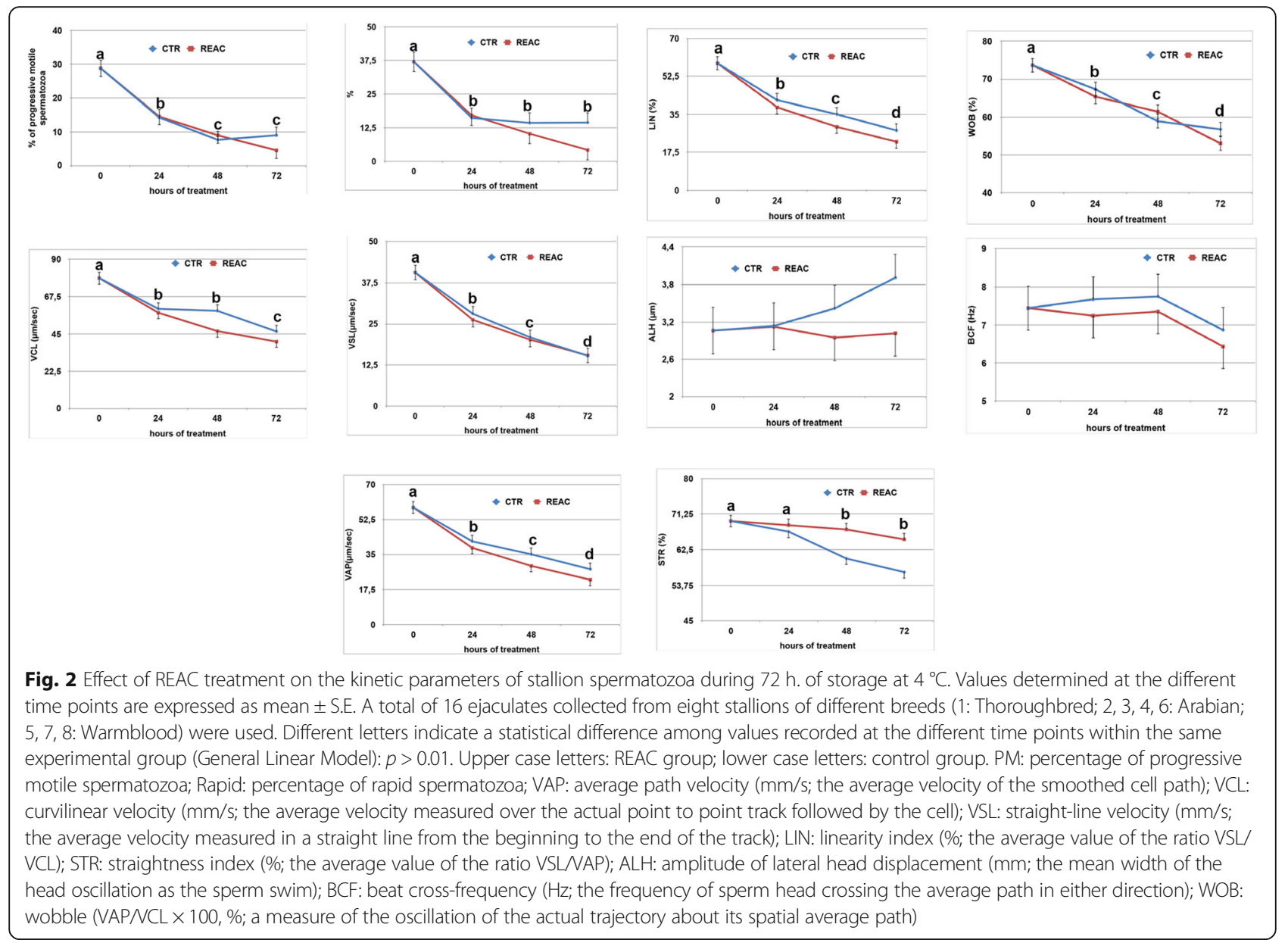

staining, and by the corresponding lower percentage of spermatozoa with damaged acrosome $(p<0.001$; Additional file 3: Figure S3, panels A and D). This difference was observed both in the mean values, and in values recorded after 48 and $72 \mathrm{~h}$. of cold storage (Fig. 3). As for the other parameters, acrosome integrity decreased over time with a similar pattern in both experimental groups. In this case, however, individual differences among the eight stallions did not reach statistical significance.

\section{DNA integrity assessment}

REAC treatment had a beneficial effect in maintaining spermatozoa DNA integrity, as evaluated by the comet assay (Fig. 4), and this protective effect was evidenced after $24 \mathrm{~h}$. of REAC treatment. A significantly smaller comet area (Additional file 4: Figure S4, panel A; $p<0.0001$ ), together with a higher percentage of DNA in the head of the spermatozoa (Additional file 4: Figure S4, panel B; $p<0.0001$ ), was found in REAC treated spermatozoa compared to untreated controls. Significant individual differences were observed among the eight stallions in both comet area and percentage of DNA in the head (Additional file 4: Figure S4, panels $\mathrm{C}$ and $\mathrm{D} ; p>0.01$ ).

\section{Oxidative status analysis}

The analysis of the oxidative status during the $72 \mathrm{~h}$. of cold storage indicated that REAC treatment had a positive effect on the enzymatic defense system of stallion spermatozoa. The mean activity of the intracellular SOD was significantly higher in REAC treated spermatozoa compared to untreated controls (Additional file 5: Figure S5, panel A; $p<0.05)$. This difference was likely linked to the marked increase in the enzyme activity observed after $48 \mathrm{~h}$. of treatment in REAC treated spermatozoa compared to untreated controls (Fig. 5, panel A; $p<0.05$ ). The increase in the intracellular antioxidant defenses of the REAC treated spermatozoa was indirectly confirmed by the significant decreased in the intracellular concentration of malondialdehyde (MDA), an end product of lipid peroxidation. While mean values did not differ between the two experimental groups, in REAC treated spermatozoa the intracellular concentration of MDA, after an initial rise at $24 \mathrm{~h}$., dropped to values significantly lower compared to the untreated controls by $72 \mathrm{~h}$. of storage (Fig. 5, panels $\mathrm{B}$ and $\mathrm{F} ; p<0.001)$. Both SOD and MDA intracellular concentration differed significantly among the eight stallions studied. The positive effect of REAC treatment on 
a

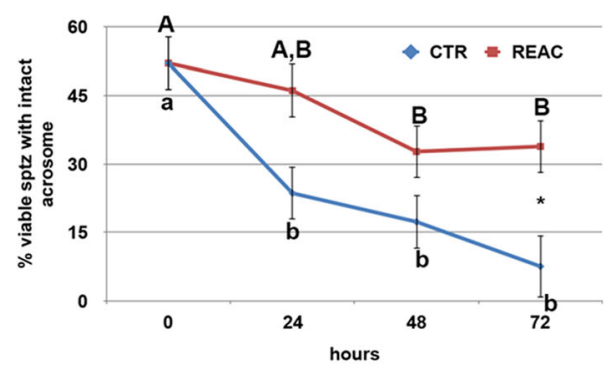

\section{b}

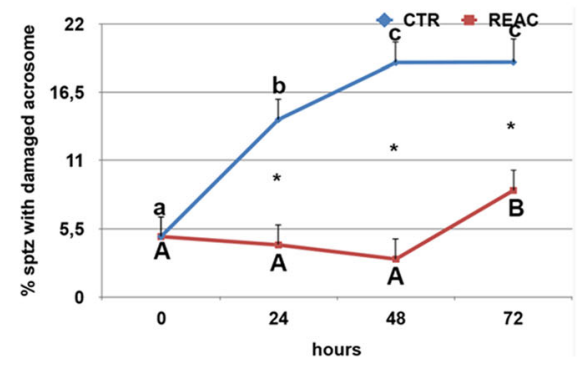

25

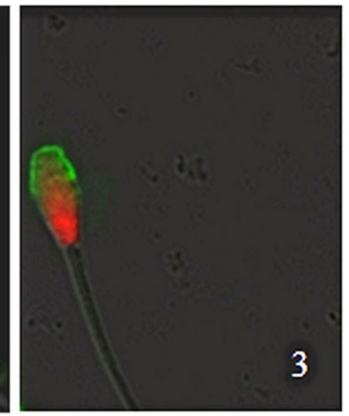

Fig. 3 Effect of REAC treatment on acrosome integrity, as evaluated by FITC-PSA staining, of stallion spermatozoa during $72 \mathrm{~h}$. of storage at $4{ }^{\circ} \mathrm{C}$. Panels $\mathbf{a}$ and $\mathbf{b}$ show the mean \pm S.E values determined at the different time points. Panel A shows the percentage of spermatozoa with intact acrosome, while panel $\mathbf{b}$ shows the percentage of spermatozoa with damaged acrosome. A total of 16 ejaculates collected from eight stallions of different breeds (1: Thoroughbred; 2, 3, 4, 6: Arabian; 5, 7, 8: Warmblood) were used. The microscopic images show a live spermatozoon with intact acrosome (1; no fluorescent staining), a live spermatozoon with damaged acrosome (2; FITC-PSA positive), and a dead spermatozoon with damaged acrosome (3; PI and FITC-PSA positive), identified using fluorescein isothiocynatelabeledPisumSativum agglutinin (FITC-PSA) and propidium iodide (PI). Asterisks indicate statistical differences between REAC treated and untreated controls (General Linear Model): $p>0.001$. Different letters indicate a statistical difference among values recorded at the different time points within the same experimental group (General Linear Model): $p>0.01$. Upper case letters: REAC group; lower case letters: control group

spermatozoa oxidative status was also supported by the higher trolox equivalent antioxidant capacity (TEAC) found in both the cellular extract (Additional file 6: Figure S6, panel $\mathrm{A} ; p<0.05$ ) and the storage media of REAC treated spermatozoa compared to untreated controls (Additional file 6: Figure S6, panel B; $p<0.0001$ ). The antioxidant capacity of the storage media rose significantly during the first $24 \mathrm{~h}$. of REAC treatment, and then remained at higher values compared to untreated controls for the entire observational period (Fig. 6, panel B; $p<0.001)$. The stallion effect influenced significantly only the TEAC values of the cellular extract (Additional file 6: Figure S6, panel C; $p>0.0001$ ), while it was not observed for values recorded in the storage media (Additional file 6: Figure S6, panel D, Additional file 7).

\section{Discussion}

The kinetic parameter significantly affected by REAC treatment was spermatozoa straightness index, i.e. the average value of the ratio straight-line velocity/average path velocity, which proved to be better preserved following REAC treatment. This parameter expresses the finalization of spermatozoa towards their fertilization task. In fact, sperm motility is essential for normal fertilization, but the orientation of motility and the motor strategy of spermatozoa are crucial features.

Among the cellular features analyzed, acrosome integrity proved to be significantly influenced by REAC treatment. The mean percentage of spermatozoa with intact acrosome was higher in REAC treated spermatozoa compared with untreated controls. Analyzing the variation of this parameter during the $72 \mathrm{~h}$. of storage, emerged that REAC treatment was effective after $48 \mathrm{~h}$. of storage, as evidenced by the higher percentages of spermatozoa with intact acrosome at 48 and $72 \mathrm{~h}$. Considering that no differences were found in semen viability during storage, the percentage of spermatozoa with damaged acrosome was higher in untreated samples compared with REAC treated ones. The acrosome is necessary for the fertilization process to occur, and a damaged acrosome results inevitably with the loss of the fertilizing ability. The preservation of acrosome membrane integrity in REAC treated spermatozoa was also accompanied by a higher DNA integrity, as revealed by the lower DNA migration after single cell electrophoresis. Even if this parameter was subjected 
a

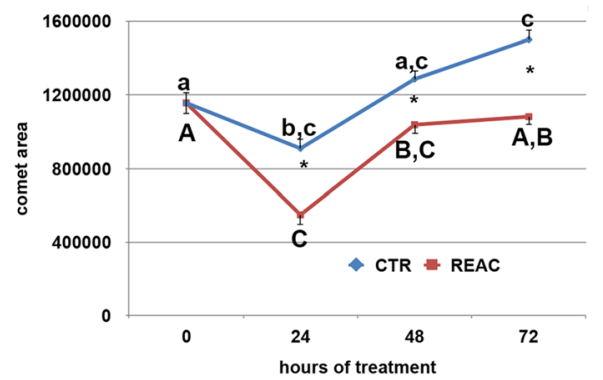

b
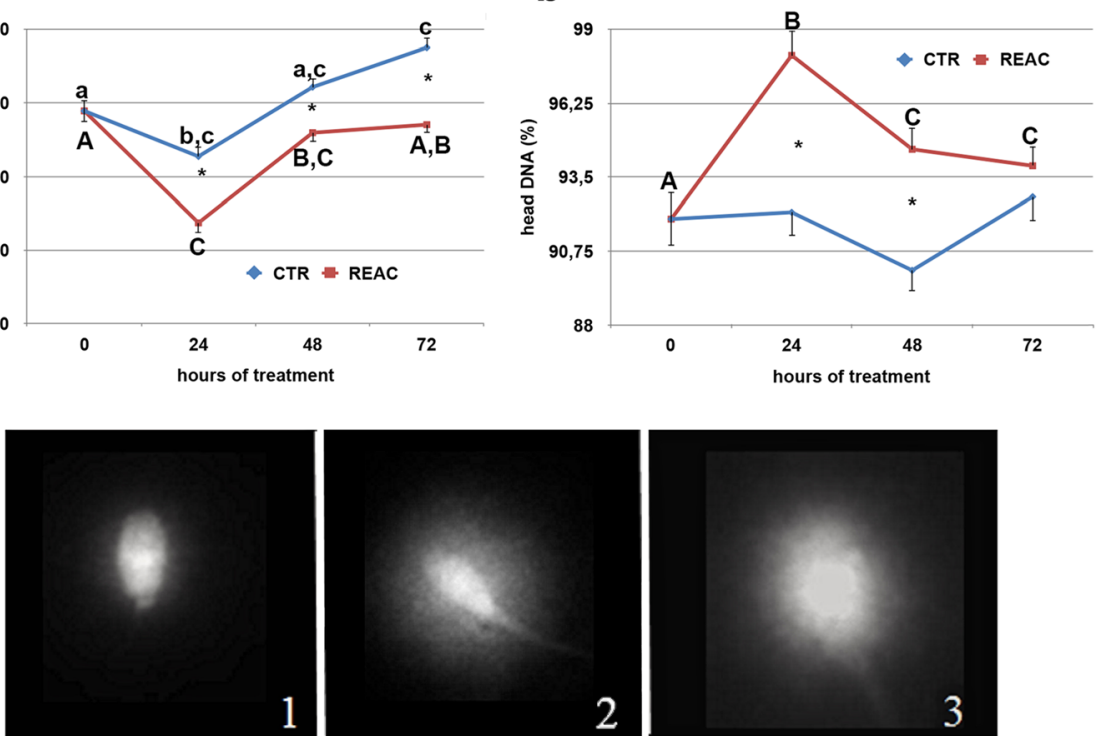

3

Fig. 4 Effect of REAC treatment on DNA integrity, as evaluated by the neutral comet assay, of stallion spermatozoa during $72 \mathrm{~h}$. of storage at $4^{\circ}$ C. Evaluated parameters included the comet area (pixels) and the percentage of DNA in the head. For each parameter, panels $\mathbf{a}$ and $\mathbf{b}$ show the mean \pm S.E values determined at the different time points in each experimental group. A total of 16 ejaculates collected from eight stallions of different breeds (1: Thoroughbred; 2, 3, 4, 6: Arabian; 5, 7, 8: Warmblood) were used. Images show representative comets following application of the neutral comet assay: (1) non-fragmented sperm nucleus; (2-3) fragmented sperm nuclei. Asterisks indicate statistical differences between REAC treated and untreated controls (General Linear Model): $p>0.01$. Different letters indicate a statistical difference among values recorded at the different time points within the same experimental group (General Linear Model): $p>0.01$. Upper case letters: REAC group; lower case letters: control group

to high inter-individual variations, comet area was significantly larger in untreated controls, the percentage of migrating DNA being higher than in REAC treated spermatozoa. Analyzing the changes in these parameters during storage revealed that REAC beneficial effect on DNA integrity became evident after $24 \mathrm{~h}$. of REAC treatment.

DNA damage in refrigerated cells may be caused by an increase in intracellular sodium concentration, which is detrimental to the nucleus and DNA [18, 19], considering that at low temperature the activity of the sodium- potassium-dependent ATPase is impaired and this can result in uptake of sodium ions from extracellular milieu. Thus, in this study we observed the beneficial effect of REAC treatment as cell polarity optimizer on the preservation of spermatozoa DNA integrity, related to its positive regulation of ion flows and cell bioelectric activity.

In addition, the preservation of both acrosome membrane and DNA integrity in REAC treated spermatozoa can be mediated by the observed effect in enhancing sperm antioxidant defenses. Antioxidants play a crucial role in minimizing oxidative damage in the spermatozoa.
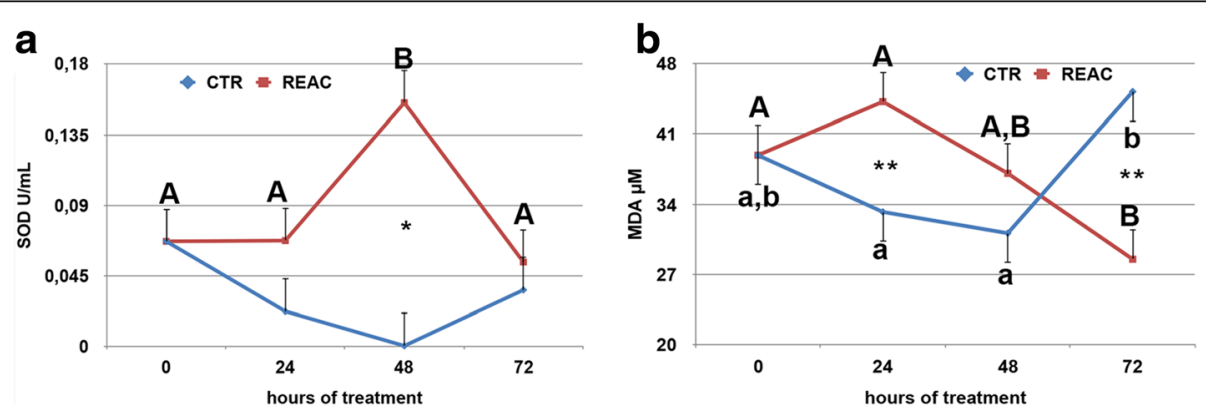

Fig. 5 Effect of REAC treatment on SOD (panel a) and MDA (panel b) intracellular concentrations of stallion spermatozoa during 72 h. of storage at $4{ }^{\circ} \mathrm{C}$. For each parameter, the mean \pm S.E values determined at the different time points are shown. A total of 16 ejaculates collected from eight stallions of different breeds (1: Thoroughbred; 2, 3, 4, 6: Arabian; 5, 7, 8: Warmblood) were used. Asterisks indicate statistical differences between REAC treated and untreated controls (General Linear Model): ${ }^{*} p>0.05 ;{ }^{* *} p<0.001$. Different letters indicate a statistical difference among values recorded at different time points within the same experimental group (General Linear Model): $p>0.01$. Upper case letters: REAC group; lower case letters: control group 

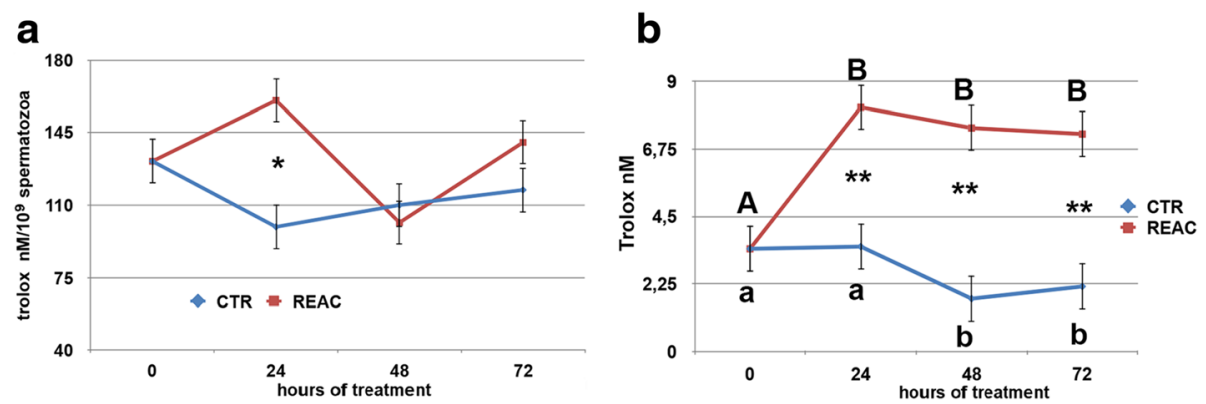

Fig. 6 Effect of REAC treatment on TEAC (trolox equivalent antioxidant capacity) in the cellular extract of stallion spermatozoa (panel a) and in the media (panel $\mathbf{b}$ ) during $72 \mathrm{~h}$. of storage at $4{ }^{\circ} \mathrm{C}$. For each parameter, panels $\mathbf{a}$ and $\mathbf{b}$ show the mean \pm S.E values determined at the different time points. A total of 16 ejaculates collected from eight stallions of different breeds (1: Thoroughbred; 2, 3, 4, 6: Arabian; 5, 7, 8: Warmblood) were used. Asterisks indicate statistical differences between REAC treated and untreated controls (General Linear Model): ${ }^{*} p>0.05 ;{ }^{* *} p<0.001$. Panel b Different letters indicate a statistical difference among values recorded at the different time points within the same experimental group (General Linear Model): $p<0.001$. Upper case letters: REAC group; lower case letters: control group

The metalloproteins SOD are an important antioxidant defense in nearly all cells exposed to oxygen. SOD, together with glutathione (GSH), is one of the most important intracellular scavenger systems spermatozoa have; it reduces the superoxide anion $\left(\mathrm{O}_{2^{-}}\right)$to hydrogen peroxide $\left(\mathrm{H}_{2} \mathrm{O}_{2}\right)$ [32]. The results of this study evidenced that SOD activity in REAC treated spermatozoa peaked at $48 \mathrm{~h}$. of storage and was significantly higher compared to untreated controls. This higher SOD activity was accompanied by a significant decrease over time in intracellular concentration of lipid peroxidase end products (MDA), whose levels at $72 \mathrm{~h}$. of storage were lower in REAC treated spermatozoa compared with untreated controls. In addition, trolox equivalent antioxidant capacity (TEAC) of both cellular extract and storage media was significantly higher in REAC treated samples compared with untreated controls.

Oxidative stress adversely changes sperm cell functions, by ultimately endangering cell survival, and causes loss of sperm motility, viability and capacity for fertilization [33].

The limited volume and restricted location of their cytoplasmic space place constraints on the availability of intracellular antioxidant enzymes in these cells. In addition, the susceptibility of spermatozoa to oxidative stress is a consequence of the superabundance of polyunsaturated fatty acids in their plasma membrane, whose presence gives the membrane the fluidity and flexibility needed to engage in membrane fusion events associated with the fertilization. Unfortunately, the presence of double bonds in these molecules makes them vulnerable to free radical attacks and the initiation of lipid peroxidation (LPO) cascade. These attacks ultimately lead to the impairment of sperm function through oxidative stress and the production of cytotoxic aldehydes, such as MDA [34]. ROS can also attack another important substrate in mammalian spermatozoa: the DNA present in the sperm nucleus [35]. Experimental studies revealed that oxidative stress results in DNA strand breaks in spermatozoa, as detected by the comet assay [36, 37]. Even if it is tightly compacted with protamines, and further stabilized by the creation of inter- and intramolecular disulphide bonds [36, 38], free radicals can still attack it, engaging in $\mathrm{H}$-abstraction reactions with the ribose unit and inducing the formation of DNA base adducts. Both of these processes greatly destabilize the DNA structure and ultimately result in the formation of DNA strand breaks [35].

For these reasons, oxidative stress of spermatozoa is a main factor in the determination of male infertility. Electron leakage from the sperm mitochondria is thought to constitute the major source of reactive oxygen species (ROS) in spermatozoa [21]. A perturbation in the electron transport chain and the loss of membrane potential are associated with a significant increase in ROS generation [21, 22]. Mitochondria have been identified as the most sensitive sperm structure to cryopreservation [39], and changes in mitochondria membrane potential have been observed in stallion spermatozoa after cooling to $4{ }^{\circ} \mathrm{C}[10]$. The exact mechanisms by which REAC treatment affected spermatozoa oxidative status still have to be elucidated. REAC treatment creates an asymmetric electric circuit in treated cells, which can modulate ion flows when these are altered and which proved to exert a positive regulation of cell bioelectric activity. Thus, we can speculate that the enhancement of sperm antioxidant defenses in REAC treated samples may be mediated by a protective effect on the preservation of mitochondria membrane potential.

\section{Conclusion}

The present study demonstrated that REAC treatment, while not affecting sperm viability and motility during refrigeration, preserves spermatozoa acrosome membrane 
and DNA integrity. This effect is likely to be mediated by the enhancement of sperm antioxidant defenses after REAC treatment, as evidenced by the higher SOD activity, the lower MDA intracellular concentration at the end of the treatment, and the higher antioxidant capacity of the cell extract and the culture medium. As suggested by other studies, any mean aimed at enhancing sperm antioxidant defenses has an important therapeutic role to play in the clinical management of male infertility [33]. Thus, this study set the basis for the use of REAC protocols aimed at enhancing the spermatozoa ability to withstand the oxidative stress related with Assisted Reproductive Technology (ART) processing.

The present study demonstrated that REAC treatment during liquid storage of chilled stallion spermatozoa preserves sperm antioxidant defenses, and as a result allows acrosome membrane and DNA integrity to be maintained longer than in untreated controls. While the loss of acrosome membrane integrity results in an impairment in spermatozoa fertilizing ability, DNA damage in the male germ line is a major contributor to infertility, miscarriage and birth defects in the offspring [40]. Thus, this technology has promising application in the in vitro treatment of sperm cells during ART processing.

\section{Additional files}

Additional file 1: Figure S1. Mean and 95,0 Bonferroni intervals of stallion spermatozoa viability, expressed as percentage of viable cells over the total cells counted. Values refer to the mean of the different values per group (control vs REAC treated, panel A) and per stallion (panel B) obtained during $72 \mathrm{~h}$. of storage at $4^{\circ} \mathrm{C}$. A total of 16 ejaculates collected from 8 stallions of different breeds (1: Thoroughbred; 2, 3, 4, 6: Arabian; 5, 7, 8 : Warmblood) were used. ${ }^{a, b, c, d}$ Different letters indicate a statistical difference among the ejaculates collected from the 8 stallions (General Linear Model): $p>0.01$. (TIF $332 \mathrm{~kb}$ )

Additional file 2: Figure S2. Effect of REAC treatment on the kinetic parameters of stallion spermatozoa during $72 \mathrm{~h}$. of storage at $4^{\circ} \mathrm{C}$. Panel $A$ and $B$ show the mean and 95,0 Bonferroni intervals per group and per stallion, respectively. A total of 16 ejaculates collected from 8 stallions of different breeds (1: Thoroughbred; 2, 3, 4, 6: Arabian; 5, 7, 8: Warmblood) were used. Panel B: a,b,c,d Different letters indicate a statistical difference among the ejaculates collected from the 8 stallions (General Linear Model): $p>0.001$. PM: percentage of progressive motile spermatozoa; Rapid: percentage of rapid spermatozoa; VAP: average path velocity $(\mathrm{mm} / \mathrm{s}$; the average velocity of the smoothed cell path); VCL: curvilinear velocity $(\mathrm{mm} / \mathrm{s}$; the average velocity measured over the actual point to point track followed by the cell); VSL: straight-line velocity $(\mathrm{mm} / \mathrm{s}$; the average velocity measured in a straight line from the beginning to the end of the track); LIN: linearity index (\%; the average value of the ratio VSL/NCL); STR: straightness index (\%; the average value of the ratio VSL/NAP); ALH: amplitude of lateral head displacement ( $\mathrm{mm}$ the mean width of the head oscillation as the sperm swim); BCF: beat cross-frequency $(\mathrm{Hz}$; the frequency of sperm head crossing the average path in either direction); WOB: wobble (VAP/VCL $\times 100, \%$; a measure of the oscillation of the actual trajectory about its spatial average path). (TIF $541 \mathrm{~kb}$ )

Additional file 3: Figure S3. Effect of REAC treatment on acrosome integrity, as evaluated by FITC-PSA staining, of stallion spermatozoa during $72 \mathrm{~h}$. of storage at $4{ }^{\circ} \mathrm{C}$. Panels $A$ and $\mathrm{D}$ show the mean and 95,0 Bonferroni intervals per group, while panels $B$ and $C$ show them per stallion. Left panels show the percentage of spermatozoa with intact acrosome, while right panels show the percentage of spermatozoa with damaged acrosome. A total of 16 ejaculates collected from 8 stallions of different breeds (1: Thoroughbred; 2, 3, 4, 6: Arabian; 5, 7, 8: Warmblood) were used. Panels A and D: Asterisks indicate statistical differences between REAC treated and untreated controls (General Linear Model): $p>0.001$. (TIF $425 \mathrm{~kb}$ )

Additional file 4: Figure S4. Effect of REAC treatment on DNA integrity, as evaluated by the neutral comet assay, of stallion spermatozoa during 72 h. of storage at $4{ }^{\circ} \mathrm{C}$. Evaluated parameters included the comet area (pixels) and the percentage of DNA in the head. For each parameter, panels $A$ and B show the mean and 95,0 Bonferroni intervals per group, while panels $C$ and $\mathrm{D}$ show them per stallion. A total of 16 ejaculates collected from 8 stallions of different breeds (1: Thoroughbred; 2, 3, 4, 6: Arabian; 5, 7, 8: Warmblood) were used. Asterisks indicate statistical differences between REAC treated and untreated controls (General Linear Model): $p>0.01$. (TIF $372 \mathrm{~kb}$ )

Additional file 5: Figure S5. Effect of REAC treatment on SOD (left side) and MDA (right side) intracellular concentrations of stallion spermatozoa during $72 \mathrm{~h}$. of storage at $4^{\circ} \mathrm{C}$. For each parameter, panels $A$ and $B$ show the mean and 95,0 Bonferroni intervals per group, while panels $C$ and $D$ show them per stallion. A total of 16 ejaculates collected from 8 stallions of different breeds (1: Thoroughbred; 2, 3, 4, 6: Arabian; 5, 7, 8: Warmblood) were used. Asterisks indicate statistical differences between REAC treated and untreated controls (General Linear Model): $p>0.05$. Different letters indicate a statistical difference among horses (General Linear Model): $p>0.01$. (TIF $397 \mathrm{~kb}$ )

Additional file 6: Figure S6. Effect of REAC treatment on TEAC (trolox equivalent antioxidant capacity) in the cellular extract of stallion spermatozoa (left side) and in the media (right side) during $72 \mathrm{~h}$. of storage at $4{ }^{\circ} \mathrm{C}$. For each parameter, panels $A$ and $B$ show the mean and 95,0 Bonferroni intervals per group, while panels $C$ and $D$ show them per stallion. A total of 16 ejaculates collected from 8 stallions of different breeds (1: Thoroughbred; 2, 3, 4, 6: Arabian; 5, 7, 8: Warmblood) were used. Asterisks indicate statistical differences between REAC treated and untreated controls (General Linear Model): ${ }^{*} p>0.05 ;{ }^{* *} p<0.0001$. Different letters indicate a statistical difference among horses and among values recorded at the different time points within the same experimental group (General Linear Model): Panel C: $p>0.0001$. Panel F: $p<0.001$. Upper case letters: REAC group; lower case letters: control group. (TIF $401 \mathrm{~kb}$ )

Additional file 7: Experimental raw data. (XLSX $351 \mathrm{~kb}$ )

\section{Abbreviations}

ALH: Lateral head displacement; ART: Assisted Reproductive Technology; BCF: Beat cross-frequency; CASA: Computer-assisted sperm analysis system; FITC-PSA: Fluorescein isothiocynatelabeled PisumSativum agglutinin; GSH: Glutathione; LIN: Linearity index; LPO: Lipid peroxidation; MDA: Malondialdehyde; PBS: Phosphate buffered saline; PI: Propidium iodide; PM: Progressive motile spermatozoa; REAC technology: Radio Electric Asymmetric Conveyor Technology; ROS: Reactive oxygen species; SOD: Superoxide dismutase; STR: Straightness index; TBA: Thiobarbituric acid; TBARS: Thiobarbituric acid reactive substances assay; TBS: Tartrate phosphate buffer; TEAC: Trolox equivalent antioxidant capacity; VAP: Average path velocity; VCL: Curvilinear velocity of spermatozoa; VSL: Straight-line velocity; WOB: Wobble index

\section{Acknowledgements}

Not applicable.

\section{Funding}

This work was funded by: Fondazione Banco di Sardegna and by Regione Autonoma della Sardegna, Fundamental Research Program, L.R. 7/2007

"Promotion of the scientific research and technological innovation in Sardinia".

\section{Availability of data and materials}

Please contact author for raw data requests.

\section{Authors' contributions}

SR and VF invented REAC technology, collaborated in conceiving the experimental design and in writing the manuscript. FB conceived and 
designed the experimental plan and wrote the manuscript. VP, SS, IC, SC, $\mathrm{DA}$ and $\mathrm{AC}$ performed the experiments and the statistical analysis and prepared figures. ESP supervised the project and wrote the manuscript. All authors read and approved the final manuscript.

\section{Competing interests}

Competing Interest as SR and VF are the inventors of the Radio Electric Asymmetric Conveyer technology. The other Authors report no conflicts of interest that could be perceived as prejudicing the impartiality of the research reported.

\section{Consent for publication}

Not applicable.

\section{Ethics approval and consent to participate}

This study followed ethical guidelines for care and use of agricultural animals for research (EC Directive 86/609/EEC for animal experiments). All experimental protocol were approved by OPBSA (Rector's decree $n^{\circ}$ 2688/2014 - 03/10/2014 $\left.n^{\circ} 24600\right)$.

\section{Author details}

${ }^{1}$ Department of Veterinary Medicine, University of Sassari, Viale Vienna 43/B, 07100 Sassari, Italy. ${ }^{2}$ AGRIS, Department of Research for Equine Reproduction, Ozieri, Sassari, Italy. ${ }^{3}$ Departments of Regenerative Medicine, Rinaldi Fontani Institute, Viale Belfiore 43, 50144 Florence, Italy. ${ }^{4}$ Research Department, Rinaldi Fontani Foundation, Viale Belfiore 43, 50144 Florence, Italy.

\section{Received: 30 November 2016 Accepted: 3 February 2017} Published online: 08 February 2017

\section{References}

1. Maioli M, Rinaldi S, Migheli R, Pigliaru G, Rocchitta G, Santaniello S, Basoli V, Castagna A, Fontani V, Ventura C, Serra PA. Neurological morphofunctional differentiation induced by REAC technology in PC12. A neuro protective model for Parkinson's disease. Sci Rep. 2015;5:10439.

2. Maioli M, Rinaldi S, Pigliaru G, Santaniello S, Basoli V, Castagna A, Fontani V, Ventura C. REAC technology and hyaluron synthase 2, an interesting network to slow down stem cell senescence. Sci Rep. 2016;6:28682.

3. Maioli M, Rinaldi S, Santaniello S, Castagna A, Pigliaru G, Delitala A, Bianchi F, Tremolada C, Fontani V, Ventura C. Radioelectric asymmetric conveyed fields and human adipose-derived stem cells obtained with a nonenzymatic method and device: a novel approach to multipotency. Cell Transplant. 2014;23:1489-500.

4. Maioli M, Rinaldi S, Santaniello S, Castagna A, Pigliaru G, Delitala A, Lotti Margotti M, Bagella L, Fontani V, Ventura C. Anti-senescence efficacy of radio-electric asymmetric conveyer technology. Age. 2013.

5. Maioli M, Rinaldi S, Santaniello S, Castagna A, Pigliaru G, Gualini S, Cavallini C, Fontani V, Ventura C. Radio electric conveyed fields directly reprogram human dermal skin fibroblasts toward cardiac, neuronal, and skeletal muscle-like lineages. Cell Transplant. 2013;22:1227-35.

6. Maioli M, Rinaldi S, Santaniello S, Castagna A, Pigliaru G, Gualini S, Fontani V, Ventura C. Radiofrequency energy loop primes cardiac, neuronal, and skeletal muscle differentiation in mouse embryonic stem cells: a new tool for improving tissue regeneration. Cell Transplant. 2012;21:1225-33.

7. Rinaldi S, Maioli M, Pigliaru G, Castagna A, Santaniello S, Basoli V, Fontani V, Ventura C. Stem cell senescence. Effects of REAC technology on telomeraseindependent and telomerase-dependent pathways. Sci Rep. 2014;4:6373.

8. Rinaldi S, Maioli M, Santaniello S, Castagna A, Pigliaru G, Gualini S, Margotti ML, Carta A, Fontani V, Ventura C. Regenerative treatment using a radioelectric asymmetric conveyor as a novel tool in antiaging medicine: an in vitro betagalactosidase study. Clin Interv Aging. 2012;7:191-4.

9. Flesch FM, Gadella BM. Dynamics of the mammalian sperm plasma membrane in the process of fertilization. Biochim Biophys Acta. 2000;1469:197-235.

10. Ortega-Ferrusola C, Sotillo-Galán Y, Varela-Fernández E, Gallardo-Bolaños JM, Muriel A, González-Fernández L, Tapia JA, Peña FJ. Detection of "apoptosis-like" changes during the cryopreservation process in equine sperm. J Androl. 2008:29:213-21.

11. Brogan PT, Beitsma M, Henning H, Gadella BM, Stout TAE. Liquid storage of equine semen: assessing the effect of D-penicillamine on longevity of ejaculated and epididymal stallion sperm. Anim Reprod Sci. 2015;159:155-62.
12. Ortega Ferrusola C, Gonzalez Fernandez L, Morrell JM, Salazar Sandoval C, Macias Garcia B, Rodriguez-Martinez H, Tapia JA, Pena FJ. Lipid peroxidation, assessed with BODIPY-C11, increases after cryopreservation of stallion spermatozoa, is stallion-dependent and is related to apoptotic-like changes. Reproduction. 2009;138:55-63.

13. Almeida J, Ball BA. Effect of alpha-tocopherol and tocopherol succinate on lipid peroxidation in equine spermatozoa. Anim Reprod Sci. 2005;87:321-37.

14. Ortega Ferrusola C, Gonzalez Fernandez L, Macias Garcia B, Salazar-Sandoval C, Morillo Rodriguez A, Rodriguez Martinez H, Tapia JA, Pena FJ. Effect of cryopreservation on nitric oxide production by stallion spermatozoa. Biol Reprod. 2009:81:1106-11.

15. Ball BA, Vo AT, Baumber J. Generation of reactive oxygen species by equine spermatozoa. Am J Vet Res. 2001;62:508-15.

16. Aitken RJ, Lambourne S, Gibb Z. The John Hughes Memorial Lecture: aspects of sperm physiology - oxidative stress and the functionality of stallion spermatozoa. J Equine Vet. 2014;34:17-27.

17. Riel JM, Yamauchi Y, Huang TT, Grove J, Ward MA. Short-term storage of human spermatozoa in electrolyte-free medium without freezing maintains sperm chromatin integrity better than cryopreservation. Biol Reprod. 2011;85:536-47.

18. Suzuki K, Yanagida K, Yanagimachi R. Comparison of the media for isolation and storage of round spermatid nuclei before intracytoplasmic injection. J Assist Reprod Genet. 1998;15:154-7.

19. Tateno H, Kimura Y, Yanagimachi R. Sonication per se is not as deleterious to sperm chromosomes as previously inferred. Biol Reprod. 2000;63:341-6.

20. Levin M. Endogenous bioelectrical networks store non-genetic patterning information during development and regeneration. J Physiol. 2014;592:2295-305.

21. Koppers AJ, De luliis GN, Finnie JM, McLaughlin EA, Aitken RJ. Significance of mitochondrial reactive oxygen species in the generation of oxidative stress in spermatozoa. J Clin Endocrinol Metab. 2008;93:3199-207.

22. Burlaka A, Selyuk M, Gafurov M, Lukin S, Potaskalova V, Sidorik E. Changes in mitochondrial functioning with electromagnetic radiation of ultra high frequency as revealed by electron paramagnetic resonance methods. Int J Radiat Biol. 2014;90:357-62.

23. Collodel G, Rinaldi S, Moretti E, Fontani V, Castagna A, lacoponi F, Terzuoli G, Cubeddu F, Careddu GM, Cossu I, Passino ES. The effect of radio electric asymmetric conveyer treatment on sperm parameters of subfertile stallions: a pilot study. Reprod Biol. 2012;12:277-84.

24. Cibrian-Uhalte E, Abdelilah-Seyfried S. Cell Polarity. In: Encyclopedic Reference of Genomics and Proteomics in Molecular Medicine. Berlin: Springer Berlin Heidelberg; 2006. p. 237-43.

25. Pintado B, de la Fuente J, Roldan ER. Permeability of boar and bull spermatozoa to the nucleic acid stains propidium iodide or Hoechst 33258 , or to eosin: accuracy in the assessment of cell viability. J Reprod Fertil. 2000;118:145-52.

26. Lewis SE, Agbaje IM. Using the alkaline comet assay in prognostic tests for male infertility and assisted reproductive technology outcomes. Mutagenesis. 2008;23:163-70.

27. Sakkas D, Moffatt O, Manicardi GC, Mariethoz E, Tarozzi N, Bizzaro D. Nature of DNA damage in ejaculated human spermatozoa and the possible involvement of apoptosis. Biol Reprod. 2002;66:1061-7.

28. Ukeda H, Maeda S, Ishii T, Sawamura M. Spectrophotometric assay for superoxide dismutase based on tetrazolium salt 3'-1-(phenylamino)-carbonyl-3, 4-tetrazolium]-bis(4-methoxy-6-nitro)benzenesulfonic acid hydrate reduction by xanthine-xanthine oxidase. Anal Biochem. 1997;251:206-9.

29. Spanier AM, Traylor RD. A rapid, direct chemical assay for the quantitative determination of thiobarbituric acid reactive substances in raw, cooked, and cooked/stored muscle foods. J Muscle Foods. 1991;2:165-76.

30. Re R, Pellegrini N, Proteggente A, Pannala A, Yang M, Rice-Evans C. Antioxidant activity applying an improved ABTS radical cation decolorization assay. Free Radic Biol Med. 1999;26:1231-7.

31. Lewinska A, Wnuk M, Slota E, Bartosz G. Total anti-oxidant capacity of cell culture media. Clin Exp Pharmacol Physiol. 2007;34:781-6.

32. Aitken RJ, Baker MA. Reactive oxygen species generation by human spermatozoa: a continuing enigma. Int J Androl. 2002;25:191-4.

33. Aitken RJ, Jones KT, Robertson SA. Reactive oxygen species and sperm function-in sickness and in health. J Androl. 2012;33:1096-106.

34. Nichi M, Goovaerts IG, Cortada CN, Barnabe VH, De Clercq JB, Bols PE. Roles of lipid peroxidation and cytoplasmic droplets on in vitro fertilization capacity of sperm collected from bovine epididymides stored at 4 and 34 degrees C. Theriogenology. 2007;67:334-40.

35. Aitken RJ, De luliis GN. On the possible origins of DNA damage in human spermatozoa. Mol Hum Reprod. 2010;16:3-13. 
36. Sawyer DE, Mercer BG, Wiklendt AM, Aitken RJ. Quantitative analysis of gene-specific DNA damage in human spermatozoa. Mutat Res. 2003:529:21-34

37. Li Z, Yang J, Huang H. Oxidative stress induces H2AX phosphorylation in human spermatozoa. FEBS Lett. 2006;580:6161-8.

38. Bennetts LE, Aitken RJ. A comparative study of oxidative DNA damage in mammalian spermatozoa. Mol Reprod Dev. 2005;71:77-87.

39. Peña FJ, Johannisson A, Wallgren M, Rodriguez Martinez H. Antioxidant supplementation in vitro improves boar sperm motility and mitochondrial membrane potential after cryopreservation of different fractions of the ejaculate. Anim Reprod Sci. 2003;78:85-98.

40. Aitken RJ, Bronson R, Smith TB, De luliis GN. The source and significance of DNA damage in human spermatozoa; a commentary on diagnostic strategies and straw man fallacies. Mol Hum Reprod. 2013;19:475-85.

Submit your next manuscript to BioMed Central and we will help you at every step:

- We accept pre-submission inquiries

- Our selector tool helps you to find the most relevant journal

- We provide round the clock customer support

- Convenient online submission

- Thorough peer review

- Inclusion in PubMed and all major indexing services

- Maximum visibility for your research

Submit your manuscript at www.biomedcentral.com/submit
Biomed Central 